\title{
Recent Pandemic Outbreak in India- Source and Management Strategy
}

\author{
Dr. Gajanan Nagare \\ Department of Biomedical Engineering \\ Vidyalankar Institute of Technology \\ Mumbai, India
}

\author{
Mr. Heramb Gupta \\ Department of Biomedical Engineering \\ Vidyalankar Institute of Technology \\ Mumbai, India
}

\author{
Ms. Jinal Darji \\ Department of Biomedical Engineering \\ Vidyalankar Institute of Technology \\ Mumbai, India
}

\begin{abstract}
Everywhere in the world there is a big well-being emergency that began in China and was also massive allinclusive. The virus named the novel coronavirus (nCovid-19) and its spread is a great threat. COVID-19 is a worldwide devastating pandemic. The world is faced with many problems such as dissemination prevention, hospital patient care, incubation analysis, and the successful action required to tackle this pandemic. The first step to finding an answer is to find out where it started and how it started. This study offers a brief overview of the spread of the first 50 cases in India and specific events that were super propagators. It utilizes the epidemic curve to graph the initiation and further growth of the COVID19. It also encompasses various steps taken together to control the transmission of the infection. The efficacy of social distancing and quarantine has been studied and the incubation duration reported. The successful Kerala government model has been reported to curb the propagation, mainly because of experience with the Nipah Virus in the past. The study provides a fundamental process-the steps for setting up a medical facility for COVID-19 and describes the way the treatment of any patient or suspected case can be carried out.
\end{abstract} Origin

Keywords-Coronavirus, India, Incubation, Kerela, Hospital,

\section{INTRODUCTION}

A pandemic is termed as an outbreak of a disease around the world. Pandemics are primarily illnesses caused by human-to-human infection transmission [1]. A pandemic is an epidemic of which affects many people from different regions and countries around the world. The global COVID-19 pandemic is one after several pandemics, including plague, influenza and cholera. This novel Coronavirus has similar characteristics to that of bats which had been transmitted to humans through pangolins or snakes and which have subsequently been transmitted from humans to humans [2]. The symptoms were similar to those of Pneumonia. As a contact-based disease, the spread of this virus was enormous. The first case was confirmed in India on 30 January 2020. Over time, the number of cases in India began to show an increase and began to show different types of transmission over different time intervals. Coronaviruses (CoVs) are a major group of viruses that mostly affect humans through the zoonotic transmission. In the last two decades, this is the third instance of the development of a novel coronavirus following severe acute respiratory syndrome (SARS) in 2003 and coronavirus respiratory syndrome in the Middle East (MERSCoV) in 2012 [3]. Covid-19, the third epidemic that resembles previous epidemics, is one of the biggest concerns in the world. Many COVID-19 cases have occurred in most countries around the world since December 2019. People with medical problems, low immunity, old age, and lung-related health issues are more likely to experience COVID-19. The symptoms of Covid-19 are cough, cold, breathing problems very similar to flu, although the mutational behavior of COVID-19 shows many different symptoms. Various precautions have been taken around the world to prevent the spread of this virus and thus to consider various measures that will help doctors and the government to manage the pandemic.

\section{ORIGIN OF THE VIRUS}

In December 2019, people in Wuhan, the capital city of Hubei province of China started going to local hospitals with severe pneumonia of unknown causes. Many of the cases had a common exposure to the Wuhan wholesale seafood market. On 31 December 2019, China notified the outbreak to the World Health Organization. Later on, 12 January 2020, it was confirmed by the World Health Organization (WHO) that a novel coronavirus was the cause of a respiratory illness in a cluster of people in Wuhan City. On 30 January 2020, Director-General WHO declared that the outbreak of novel coronavirus (2019-nCoV) constitutes a Public Health Emergency of International Concern (PHEIC) as per the advice of International Health Regulations (IHR) Emergency Committee [4]. As of 31 January 2020, a total of 9720 confirmed cases and 213 deaths have been reported in China. Outside of China, nineteen countries have reported a total of One Hundred and Six confirmed cases, most with travel history from China. These countries are Australia, Cambodia, Canada, Finland, France, Germany, India, Italy, Japan, Malaysia, Nepal, Philippines, Singapore, South Korea, Sri Lanka, Thailand, UAE, USA, and Vietnam [4].

\section{COVID-19 OUTBREAKS IN INDIA}

A laboratory confirmed case of COVID-19 was reported in Kerala that was a student who returned from Wuhan. From then the travel advisories were issued which included 
screening of people who were returning from china and who had symptoms such as cough, cold, fever and also nonessential travel to China was prohibited. India evacuated 324 people on 1 February 2020 and 323 people on February 2020 from Wuhan region and were quarantined for 14 days and tested negative [5]. Many actions were taken in order to prevent transmission in India by screening the people on the airport itself and advising those people to stay self-quarantined irrespective of the symptoms and the suspect cases were in facility isolation. Also 112 people from Wuhan which were quarantined at camp managed by army and 124 more people were evacuated from Japan which were kept isolated on the on the Diamond Princess Cruise ship at Yokohama before bringing back to India [6]. In February 2020 there were only 3 cases but in March the cases started to increase and schools and colleges in India were ordered to be closed starting from 5 March in Delhi and later on in other states too.

All states and districts started taking some voluntary actions in order to stop the spread that is the celebrations publicly were prohibited and all the public places were made to be closed. The malls, cinema halls and all such places were made to be shut down and there were no permissions granted for any of the public celebrations. The surveillance system in India included many major changes including all the passengers coming from all the countries were screened on the Airport and the count of the airports to be screened increased to 30 and all those travelling from different countries were made to stay under isolation for about 14 days and all the information regarding passengers was tracked and along with that there was cluster tracking which helped India to reduce the spread at some extent. Later-on apart from the evacuation which happened in the February month there was lot of evacuation happening from different countries in month of March too but their isolation was well taken care of. In India slowly and steadily all places started to shut down. WHO declared the Novel Coronavirus Disease (COVID-19) outbreak as a pandemic.

On 22 March 2020, Indian citizens observed 'Janata Curfew' starting from 7:00 AM till 9:00 PM. Later-on a nationwide lockdown was declared till 31 March 2020 with only essential services opened and no landing of fights and railways to be closed. The Indian government had announced a countrywide lockdown for three weeks starting at midnight on 24 March to slow the spread of COVID-19 as the number of people testing positive in the country reached 563. Most of these people had contact with travelers returning from countries affected by COVID-19, a few had been contracted locally, suggesting that community transmission may have begun in some Indian states. Indian Government announced that all government offices would be shut for 21 days, except essential services such as the fire service, police, and hospitals. There were many hospitals and testing labs assigned and sample testing was increased considerably. On 27 and 28 of March 2020 there came forward drastic increase in the cases which were the result of public functions which took place in the starting or mid of march. After this there was an increase in COVID-19 infected patients nationwide. There was a call for 21 days lockdown which had stringent rules, Social distancing had to be highly practiced. The reason behind the slow growth of patients was the social distancing which was practiced very strictly. Also, in many places almost throughout India Section 144 of Criminal Procedure Code (CrPC) was imposed which restricted a person or a group of people residing in a particular area to visit a certain place or area. As the end of the lockdown period approached, several state governments recommended extending the lockdown. The State Governments namely Odisha, Punjab, Maharashtra, and West Bengal governments had extended the state lockdowns to 30 April 2020. At the end day of the 21day of the lockdown the lockdown had been extended up to 3 May 2020. On 14 April 2020, the lockdown 1.0 was again extended till 3 May, with some sort of relaxations from 20 April 2020 for the places that have been able to reduce the spread. And for this lockdown 2.0, many guidelines had been revised. After that there was an extension in lock down until 14 May 2020 which was lockdown 3.0, during this lock down the country was divided into three zones (Green, Orange and Red) depending on the number of cases and therefore few concessions in lockdown were granted. But considering the increased cases later there was a lockdown until 30 May 2020 which was a lockdown 4.0.

\section{FIRST 50 CASES IN INDIA}

The first case in India was found on 30 January 2020 in Thrissur Kerala; the patient had a travel history to Wuhan. Later, on 2nd and 3rd of February 2020, two more confirmed cases in Kerala were reported. These two COVID-19 confirmed cases came into light one in Delhi (Mayur Vihar) who had a travel history to Italy and the other in Hyderabad who had a travel history to Dubai. The person in Delhi (Mayur Vihar) proved to be super infectious and infected 6 people which were confirmed positive in Agra on 4 March 2020, which further infected a person in Uttar Pradesh who was confirmed positive on 9 March 2020. A 63-year-old Italian tourist confirmed positive for the novel COVID-19 in Jaipur on 3 March 2020 which infected 16 more people and confirmed positive on 4 March 2020, one of which was the wife of 63-year-olds and 14 others from the same Italian tourist group along with one Indian driver who drove for them. On 5 March 2020, two more cases came forward one was from Delhi (NCR) and had a travel history to Iran and another case was an employee of the firm in Gurgaon who had a travel history to Italy was confirmed on 9 March 2020. On 6 March 2020, one patient was confirmed in Delhi who travelled to Thailand and Malaysia. On 7 March 2020, two more cases were confirmed one in Ladakh and the other in Chennai who had travelled to Iran and Oman respectively. On 8 March 2020, five more cases were confirmed in Kerala all from the same family among which 3 had travelled to Italy and the other two contracted it from them. On 10 March 2020 six more cases were found which includes 2 in Pune and 4 in Karnataka who had travelled to Dubai and the United States. As of 18 April 2020, there have been 2,160,207 confirmed cases of COVID-19 with 85678 new cases (in the last 24 hours) in the world and 25,291 confirmed cases of COVID-19 with 1731 new cases in the South-East Asia Region [7].

\section{EPIDEMIC CURVE}

Epidemic curve is a graphical representation of the incidences of a disease plotted over time and is useful for inferring the magnitude, incubation duration, and other 
attributes of an epidemic [8]. The epidemic curve is the number of reported cases by date of symptom onset [9]. This curve is obtained by plotting the quantity of cases of the disease outbreak over a period. The curve indicates mode of spread of an outbreak. The transmission can occur within the following ways:

(i) Point source: during a source outbreak, persons are exposed over a short time to the identical source, like one meal or an occasion. the quantity of cases rises rapidly to a peak and falls gradually. The bulk of cases occur within one period of time of the disease.

(ii) Continuous common source: during a continuous common-source outbreak, persons are exposed to the identical source, but exposure is prolonged over days, weeks, or longer. The epi curve rises gradually and might plateau.

(iii) Person-to-person spread (propagation): during a propagated outbreak, there's no common source because the outbreak spreads from person-to-person. The graph will assume the classic epi curve shape of progressively taller peaks, each being one period of time apart. The "incubation period" means the time between catching the virus and setting out to have symptoms of the disease. Most estimates of the period of time for COVID-19 range from 1-14 days, most typically around five days [10].

\section{METHODS}

There have been several speculations about the number of cases of COVID-19, worldwide. Several sources talk about the cases in a particular country or a region. After considering the cases in the country of the Republic of India, within the period of 15 February 2020 to 18 April 2020, many sources talk about the case count. The case counts shows of the sources which were used: (1) World Health Organisation United Nations, (2) Statista - Global Business Data Platform, (3) Worldometer, (4) India COVID-19 Tracker- A Crowdsourced Initiative, (5) Ministry of Health and Family Welfare, Government of India - Official Public Service Announcement on Coronavirus from the Government, (6) Trading View Website COVID 19 Stats [15-20]. The average of the total number of cases per-day is taken for further calculations and plotting of the epidemic curve. Further, the number of new cases per-day was determined from this average. This was done by subtracting the total average of cases the day before from the total average cases of the day of which the number of new cases has been identified.

\section{DISCUSSION}

The shape of an epi curve seldom fits exactly with either of these descriptions. For propagated outbreaks, the shape may demonstrate overlapping waves of cases that may diminish more slowly over time by obscuring subsequent peaks and peaks. General observation of an outbreak 's mode of spread may therefore be made from its epi curve [11]. Hence, the plot of the epidemic curves presents the number of cases of each source and is shown in Fig 1. It has been observed that the number of cases per day has a constant number of three and it is the same for all the six references till 1 March 2020. After the number of cases starts increasing with time, there are slight variations in numbers across the datasets. The number of cases follows a general trend and the nature of the increase in the number per day is similar. The figure 2 is the epidemic curve of the average of the total number of cases per day. Analyzing the number of new cases, every day is an important factor to determine the growth and spread of COVID-19. The obtained number of new cases per day by using the total average number of cases in a day is presented in the bar graph (Fig 3).

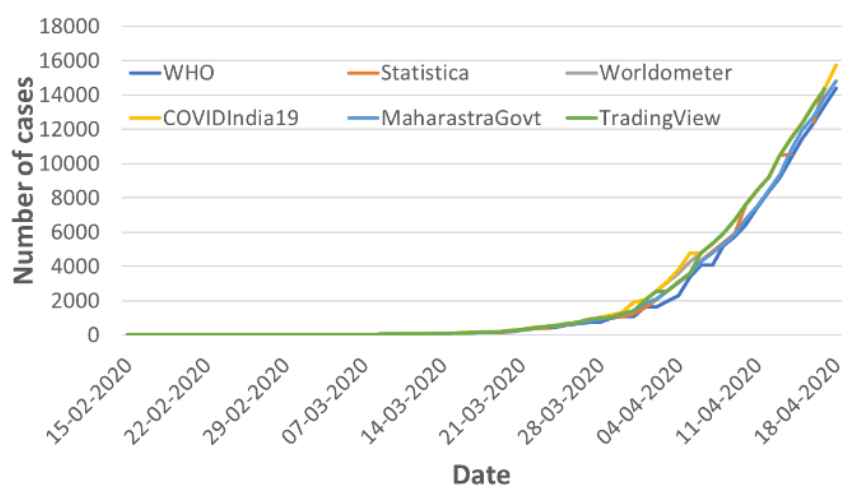

Fig 1: COVID-19 Outbreak in India by Different Sources

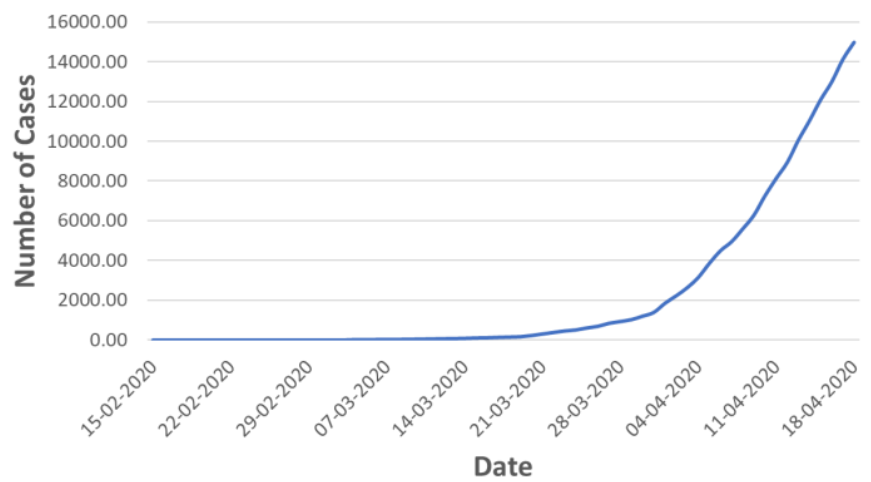

Fig 2:COVID-9 Average growth across country

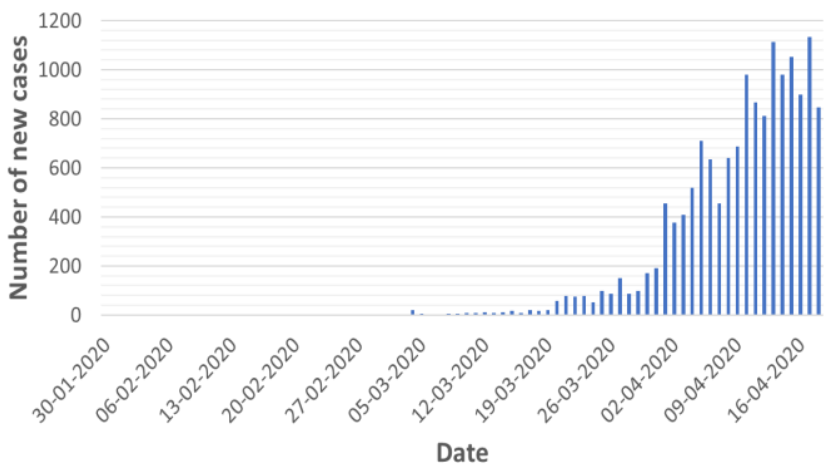

Fig 3: Spread of COVID 19 in India getting peak of infected cases

Followings are the observations and findings from the epidemic curve:

(i) There was a spike in the number of new cases (average of 18.67 cases) on 4 March 2020 as compared to the previous day 3 March 2020 (1 case) and 5 March 2020 (5.33 cases). The incident which caused these cases was contained very well as a result of which the number went back to the normal level. 
(ii) The number of new cases per day almost doubled on 15 March 2020 (18.00 cases) as compared to the previous days 14 March 2020 (11.33 cases) and 13 March 2020 (7.00 cases). In the following days the number of new cases then rose to match this level, 16 March 2020 (8.33 cases), 17 March 2020 (20.8333 cases) and 18 March 2020 (17.47 cases).

(iii) The number of new cases per day at least tripled on 20 March 2020 (58.833 cases) as compared to the previous day's 19 March 2020 (18.87 cases) and 18 March 2020 (14.47 cases) and stayed in this range till the 29 March where the cases went to as high as 98.8333 cases per day (25 March 2020).

(iv) There was a spike in the number of new cases (149.00 cases) on 27 March 2020 as compared to the previous day's 26 March 2020 (85.17 cases) and 28 March 2020 (87.83 cases). This was because A Sikh preacher that returned from travel to Italy and Germany, carrying the virus, turned into "super spreader" by attending a Sikh festival in Anandpur Sahib from 10-12 March.

(v) The number of new cases per day at almost tripled on 1 April 2020 (455.33 cases) as compared to the previous day's 31 March 2020 (190.33 cases) and 30 March 2020 (171.33 cases). After this day the new number of cases per day has increased almost every day.

(vi) There was a spike in the number of new cases (149.00 cases) on 27 March 2020 as compared to the previous day 26 March 2020 (85.17 cases) and 28 March 2020 (87.83 cases).

(vii) There was a sudden spike in the number of new cases on 1 April 2020 (455.33 cases). After this, the curve started to follow a trend where the number of new cases dropped for a day or two followed by an increase in the number of cases till it reached a new spike. After this, the cycle of decrease and increase of cases has continued till 18 April 2020.

After the sudden rise in the number of cases on 1 April 2020 the curve started showing characteristics of a propagated outbreak. The difference of time between two successive peaks gives us information about the incubation period of COVID-19 in India. The difference is as (1) 1 April 2020 to 5 April 2020: 4 days, (2) 5 April 2020 to 10 April 2020: 5 days, (3) 10 April 2020 to 13 April 2020: 3 days, and, (4) 13 April 2020 to 17 April 2020: 4 days. The study showed the average incubation period for India is 4 days.

\section{MANAGEMENT PLAN FOR PEOPLE WITH COVID-19 SYMPTOMS}

It is essential to practice precautionary measures to prevent transmission and reduce the risk of infection from person to person. While considering the person who has symptoms which resembles to be that of COVID-19 there are various plans to be considered while managing the patients so that the disease doesn't spread further and we get success in breaking the chain of spread. It is important that all physicians likely to handle the cases should know about the principles of management of these people keeping in mind limited resources such as facilities for test, places for isolation, Limited medical facilities and most importantly there is no

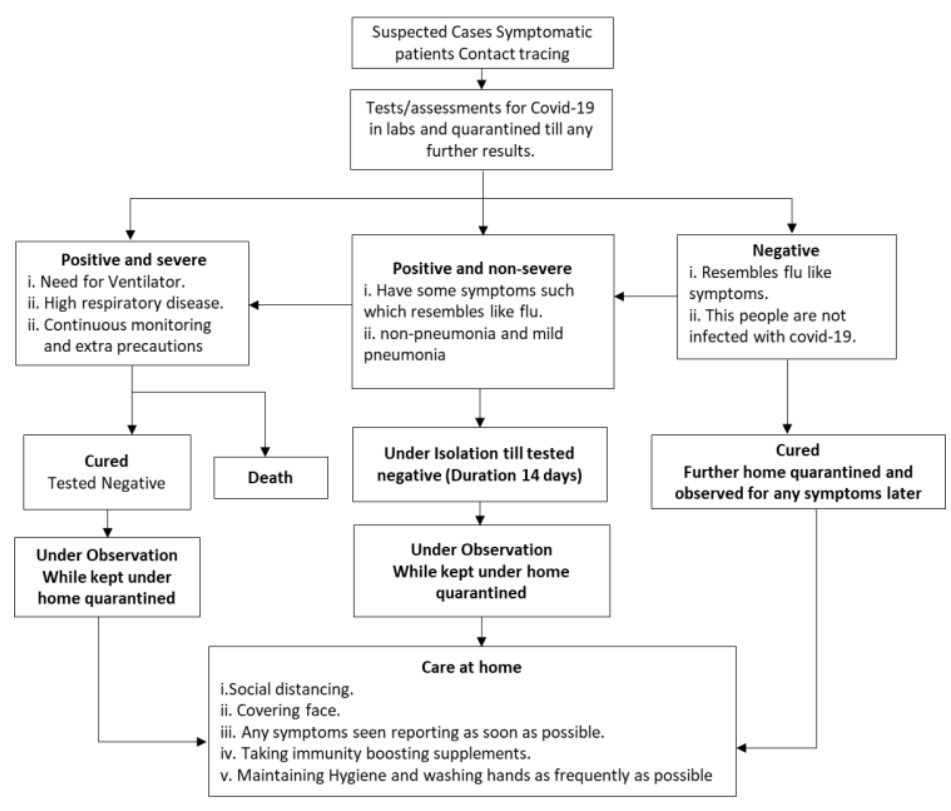

Fig 4: Management plan for people with symptoms for COVID-19

vaccine or drug in order to cure the disease. The flow of steps to manage the symptomatic patient are given in Fig 4.

\section{THE KERELA MODEL}

In 2018, the state had dealt with an outbreak of Nipah, a brain-damaging virus that, like the coronavirus, had originated in bats and transferred to humans. Nipah viral disease outbreak had been reported in Kozhikode district, northern Kerala, India and the fruit bats had been identified as the source of the outbreak [20]. This disease was considered to be very new and there was no such strong evidence related to it. Intensive measures were initiated by the state with the help of central government health authorities including the National Centre for Disease Control, National Institute of Epidemiology, Indian Council of Medical Research (ICMR), and experts from AIIMS, New Delhi. Initiatives taken to prevent the spread was majorly contact tracing and quarantining. Strict isolation precautions were followed in order to prevent the spread and to control the epidemic also strict measures were taken in order to prevent the droplet infections. At the community level, a special task force was set up to re-ensure early suspicion and detection followed by isolation, treatment, and surveillance of confirmed cases [21]. The utmost care was taken by the government and medical facilities resulted in early suspicion of the deadly epidemic in a span of 12 days. The World Health Organization (WHO)

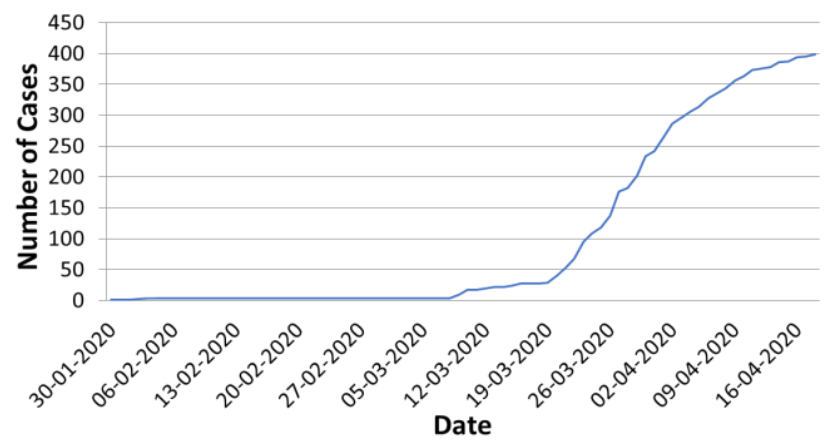

Fig 5: Number of Cases in Kerala 
termed Kerala's handling of the outbreak a "success story". Kerala in spite of technical shortcomings was able to contain a potential disaster.

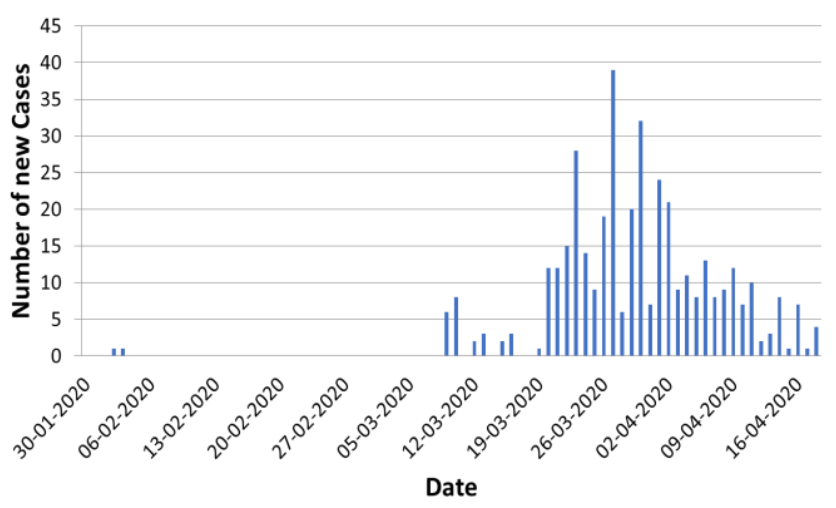

Fig 6: Number of new cases in Kerala

The epidemic curve shows how the number of cases varied with time (Table 1), we see that the Kerala Government was successful to begin the flattening of the curve by 18 April 2020 (Fig. 5) and the new number of cases everyday also decreased (Fig. 6). As a result of this a number of steps that were implemented in the state of Kerala can be implemented across the entire country.

(i) Quarantine measures: Kerala is the only state that mandates 28 days of home quarantine, while the national guidelines have been 14 days for those who return from countries affected by COVID-19. After permitting International travel over time, all travelers returning from countries with a travel history from a country where there are positive cases to be mandated a home quarantine of 28 days. Those travelers who are coming from a country with lesser cases to be mandated a quarantine of 14 days for safety purposes. This Quarantine to be mandated with the means of the Aarogya Setu App.

(ii) Care Homes: In Kerala COVID-19 care homes were set up near the international airports. These care homes were set up in hospitals and even prison wards along with with isolation beds. Private hospitals and buildings that were abandoned and had rooms with attached bathrooms were taken over by the district administration and converted to care centers.

(iii) Tele Counselling Service: A 24X7 Disha call center was set up to counsel quarantined families on responsible behavior and to improve their mental health. This step is very necessary to be adapted all over India to ensure the mental stability of all the citizens during the lockdown. The 626 medical personnel who were employed also ran the patients through a questionnaire which was meant to assess their physical and mental health, but also to catch lies. If anyone was caught sneaking out, the police, the revenue department, and village councils were ready to act and this office also delivered groceries to those in need.

(iv) Demand and Supply: Foreseeing a growing demand for medical supplies at a time of crisis, a team of officials were entrusted to procure the necessary medical supplies such as masks, sanitizers and personal protection equipment gears. There was an enormous community participation as not only volunteer organizations and companies but also prisoners in certain districts were trained and engaged to produce masks and sanitizers. The citizens of India are facing numerous difficulties to acquire the required protective gear. These steps can be implemented to supply the demand of increasing medical supplies.

(v) Break the Chain: Under the "Break the Chain" initiative, the government had installed water taps accompanied by sanitizers at public places implementing basic hygiene measures. This initiative should be implemented all over the country especially in places where there is a lack of clean drinking water.

(vi) Transparency: It is a necessary condition for an effective response. The Chief Minister of Kerala provides an update of the scenario in the state, via a televised address, daily, in which he informs citizens about the number of new cases per day, number of patients recovered, and the number of deaths; he outlines the actions the government has taken and reinforces the importance of precautionary measures, such as physical distancing. He also informs the people of Kerala about the actions they can take, should they face any issues accessing healthcare. This level of communication ensures that the fear among people is reduced and thus acts as a confidence-building measure in the government's handling of the situation. If not at a national level this measure should be implemented by individual state governments to maintain the trust of the people and ensure cooperation.

(vii) Diverse Action Plan: The Kerala state government invited religious leaders, local bodies and civil society organizations to participate in policy design and implementation, considering its specific socio-economic conditions, including urban slum environments. It has communicated effectively in different languages to educate all, including migrants, and to prevent stigmatization of those infected, even opposing the term 'social distancing', which has caste connotations, with 'physical distancing and social solidarity'. India is a very diverse country. The population has several people from many varied backgrounds. To reach to every citizen the above-mentioned steps are very necessary for effective implementation of plans and policies.

The Government of Kerala looked at the Corona Crisis from various points of view. As we can call it the "Seven Point Plan" which includes (1) Patient Care of affected citizens, (2) Effective management of patients by quarantine and isolation measures, (3) Mental health of all citizens, (4) Maintenance of Public Hygiene, (5) Networking and effective communication, (6)Implementation of Government Rules with cooperation and monitoring, and (7) Management of increased demand of medical supplies; played an instrumental role in successfully curbing larger effects of COVID-19. Every State Government in India with the help of the Central Government should align its plan of action and policies with this Seven Point Plan to tackle the problem of COVID-19 effectively.

\section{CONCLUSION}

This new outbreak of the virus has challenged not only India's medical and public health infrastructure, but also the world as a whole. According to the analysis of the epidemic curve, done in this paper, the incubation period is found to be 4 days in this first wave of COVID-19 in India. If the first wave is successfully curbed, there is a likelihood of a second 
Table 1: COVID-19 Cases in Kerala

\begin{tabular}{|c|c|c|c|c|c|c|c|c|c|c|c|}
\hline Date & $\begin{array}{c}\text { Kerala } \\
\text { Government } \\
\text { Dash Board }\end{array}$ & $\begin{array}{l}\text { New } \\
\text { Cases }\end{array}$ & Date & $\begin{array}{c}\text { Kerala } \\
\text { Government } \\
\text { Dash Board }\end{array}$ & $\begin{array}{l}\text { New } \\
\text { Cases }\end{array}$ & Date & $\begin{array}{c}\text { Kerala } \\
\text { Government } \\
\text { Dash Board }\end{array}$ & $\begin{array}{l}\text { New } \\
\text { Cases }\end{array}$ & Date & $\begin{array}{c}\text { Kerala } \\
\text { Government } \\
\text { Dash Board }\end{array}$ & $\begin{array}{l}\text { New } \\
\text { Cases }\end{array}$ \\
\hline $30-01-2020$ & 1 & 0 & $19-02-2020$ & 3 & 0 & $10-03-2020$ & 17 & 8 & $30-03-2020$ & 234 & 32 \\
\hline $31-01-2020$ & 1 & 0 & $20-02-2020$ & 3 & 0 & $11-03-2020$ & 17 & 0 & $31-03-2020$ & 241 & 7 \\
\hline $01-02-2020$ & 1 & 0 & $21-02-2020$ & 3 & 0 & $12-03-2020$ & 19 & 2 & $01-04-2020$ & 265 & 24 \\
\hline $02-02-2020$ & 2 & 1 & $22-02-2020$ & 3 & 0 & $13-03-2020$ & 22 & 3 & $02-04-2020$ & 286 & 21 \\
\hline $03-02-2020$ & 3 & 1 & $23-02-2020$ & 3 & 0 & $14-03-2020$ & 22 & 0 & $03-04-2020$ & 295 & 9 \\
\hline $04-02-2020$ & 3 & 0 & $24-02-2020$ & 3 & 0 & $15-03-2020$ & 24 & 2 & $04-04-2020$ & 306 & 11 \\
\hline $05-02-2020$ & 3 & 0 & $25-02-2020$ & 3 & 0 & $16-03-2020$ & 27 & 3 & $05-04-2020$ & 314 & 8 \\
\hline $06-02-2020$ & 3 & 0 & $26-02-2020$ & 3 & 0 & $17-03-2020$ & 27 & 0 & $06-04-2020$ & 327 & 13 \\
\hline $07-02-2020$ & 3 & 0 & $27-02-2020$ & 3 & 0 & $18-03-2020$ & 27 & 0 & $07-04-2020$ & 335 & 8 \\
\hline $08-02-2020$ & 3 & 0 & $28-02-2020$ & 3 & 0 & $19-03-2020$ & 28 & 1 & $08-04-2020$ & 344 & 9 \\
\hline $09-02-2020$ & 3 & 0 & $29-02-2020$ & 3 & 0 & $20-03-2020$ & 40 & 12 & $09-04-2020$ & 356 & 12 \\
\hline $10-02-2020$ & 3 & 0 & $01-03-2020$ & 3 & 0 & $21-03-2020$ & 52 & 12 & $10-04-2020$ & 363 & 7 \\
\hline $11-02-2020$ & 3 & 0 & $02-03-2020$ & 3 & 0 & $22-03-2020$ & 67 & 15 & $11-04-2020$ & 373 & 10 \\
\hline $12-02-2020$ & 3 & 0 & 03-03-2020 & 3 & 0 & $23-03-2020$ & 95 & 28 & $12-04-2020$ & 375 & 2 \\
\hline $13-02-2020$ & 3 & 0 & $04-03-2020$ & 3 & 0 & $24-03-2020$ & 109 & 14 & $13-04-2020$ & 378 & 3 \\
\hline $14-02-2020$ & 3 & 0 & $05-03-2020$ & 3 & 0 & $25-03-2020$ & 118 & 9 & $14-04-2020$ & 386 & 8 \\
\hline $15-02-2020$ & 3 & 0 & $06-03-2020$ & 3 & 0 & $26-03-2020$ & 137 & 19 & $15-04-2020$ & 387 & 1 \\
\hline $16-02-2020$ & 3 & 0 & $07-03-2020$ & 3 & 0 & $27-03-2020$ & 176 & 39 & $16-04-2020$ & 394 & 7 \\
\hline $17-02-2020$ & 3 & 0 & $08-03-2020$ & 3 & 0 & $28-03-2020$ & 182 & 6 & $17-04-2020$ & 395 & 1 \\
\hline $18-02-2020$ & 3 & 0 & 09-03-2020 & 9 & 6 & $29-03-2020$ & 202 & 20 & $18-04-2020$ & 399 & 4 \\
\hline
\end{tabular}

wave of the outbreak in India. Therefore, in addition to curbing the outbreak, efforts should be made to prevent a future outbreak by implementing the seven-point plan that has proved to be

effective in controlling COVID-19 in Kerala. Lastly, our analysis suggests transmission reduction measures, as COVID-19 will stay for a considerable amount of time and the world will have to learn to adapt to certain standards that will further help us eradicate the pandemic. In India, the national lockdown has had a huge impact on the control of the spread of the virus. However, citizens should keep in mind that, once the lockdown is over, citizens will still have to avoid close contact at the individual level.

\section{REFERENCES}

[1] Qiu, W. \& Rutherford, Shannon \& Mao, A. \& Chu, Cordia. (2017) The Pandemic and its Impacts.Health, Culture and Society. 9. 1-11. 10.5195/HCS.2017.221.

[2] Singhal, T. A Review of Coronavirus Disease-2019 (COVID-19). Indian J Pediatr 87, 281-286 (2020). https://doi.org/10.1007/s12098020-03263-6.

[3] Chatterjee P, Nagi N, Agarwal A, Das B, Banerjee S, Sarkar S, Gupta N, Gangakhedkar RR. The 2019 novel coronavirus disease (COVID19) pandemic: A review of the current evidence. Indian J Med Res 2020; 151:147-59.

[4] World Health Organization. (2020, January 31).Novel Corona virus(2019-nCov) India Situation report1.

[5] "Coronovirus outbreak: People flown in from China quarantined". The Hindu. (2020, February 1) Retrieved from https://www.thehindu.com/news/national/coronavirus-outbreak-air india-flight-evacuating-324-indians-from-chinas-wuhan-land-indelhi/article30709431.ece.

[6] "India Situation Report." World Health Organization, World Health Organization, www.who.int/india/emergencies/coronavirus-disease(covid-19)/india-situation-report.

[7] World Health Organization, Coronavirus disease 2019 (COVID-19) Situtation report 89," vol. 2019, 18 April, 2020.
[8] Nsoesie EO, Beckman R, Marathe M, Lewis B. Prediction of an Epidemic Curve: A Supervised Classification Approach. Stat Commun Infect Dis. 2011;3(1):5. doi:10.2202/1948-4690.1038.

[9] Wallinga, Jacco \& Teunis, Peter. (2004). Different Epidemic Curves for Severe Acute Respiratory Syndrome Reveal Similar Impacts of Control Measures. American journal of epidemiology. 160. 509-16. 10.1093/aje/kwh255.

[10] World Health Organization, Coronavirus disease 2019 (COVID-19) Situation reports.

[11] Centre for disease control and prevention, "CDC LC Quick Learn Using an Epi Curve to Determine Mode of Spread", Retrieved from https ://www.cdc.gov/ training/QuickLearns/ epimode/Quick Learn.

[12] Rawat, Mukesh. "Coronavirus in India: Tracking Country's First 50 COVID-19 Cases; What Numbers Tell.” India Today, 12 Mar. 2020 https://www.indiatoday.in/india/story/coronavirus-in-india-trackingcountry-s-first-50-covid-19-cases-what-numbers-tell-1654468-2020 03-12.

[13] "COVID-19 Situation Reports." World Health Organization, World Health Organization, https://www.who.int/emergencies/diseases/novelcoronavirus-2019/situation-reports.

[14] "Coronavirus in India: Latest Map and Case Count." COVID https://www.covid19india.org/demographics.

[15] "Coronavirus (COVID-19) Charts and Stats." TradingView, https://in.tradingview.com/ covid19/.

[16] " India." https://www.worldometers.info/coronavirus/country/india/

[17] Diwanji, Sanika. "India: COVID-19 Cases Timeline 2020." Statista, 21 May 2020, https://www.statista.com/statistics/1104054/indiacoronavirus-covid-19-daily-confirmed-recovered-death-cases/.

[18] “\#IndiaFightsCoronaCOVID-19." MyGov.in, 3 Apr. 2020 https://www.mygov.in/covid-19/.

[19] Rachita Vora. "COVID-19 and lessons from Kerala" idronline.org, April 01，2020.Retrieve from https://idronline.org/covid-19-andlessons-from-kerala/.

[20] Chatterjee P. 2018. Nipah virus outbreak in India. Lancet. 391(10136):2200. doi:10.1016/S0140-6736(18)31252-2.

[21] Ajith Kumar AK, Anoop Kumar AS. Deadly Nipah Outbreak in Kerala: Lessons Learned for the Future. Indian J Crit Care Med. 2018;22(7):475-476. 